\title{
Akt regulation of Aven contributes to the sensitivity of cancer cells to chemotherapeutic agents
}

\author{
KYOUNG-YOUN HAN ${ }^{1}$, JEE WON HWANG ${ }^{1}$, GYU-UN BAE $^{1}$, SU-NAM KIM ${ }^{2}$ and YONG KEE KIM ${ }^{1}$ \\ ${ }^{1}$ Research Center for Cell Fate Control, College of Pharmacy, Sookmyung Women's University, Seoul 140-742; \\ ${ }^{2}$ Natural Products Research Center, Korea Institute of Science and Technology, Gangneung Institute, \\ Gangneung, Gangwon-do 210-340, Republic of Korea
}

Received February 27, 2014; Accepted November 19, 2014

DOI: $10.3892 / \mathrm{mmr} .2015 .3158$

\begin{abstract}
In the present study, it was demonstrated that the protein level of the apoptosis inhibitor Aven is regulated by the Akt signaling pathway, evidenced by the observation that Aven levels were significantly increased in MCF7 constitutively active (CA)-Akt cells and significantly inhibited following treatment with LY294002. This increase in Aven appears not to be mediated by transcriptional regulation and protein stabilization. However, the level of Aven was inversely correlated with the level of cathepsin D, which is a protease responsible for generating the C-terminal of Aven, $\Delta \mathrm{N}$-Aven, indicating that the level of Aven appears to be regulated by cathepsin D activity. It has previously been reported that $\Delta \mathrm{N}$-Aven is the active form of Aven, which functions as an anti-apoptotic molecule. Notably, low levels of $\triangle \mathrm{N}$-Aven were detected in MCF7 CA-Akt cells, which were more sensitive to anticancer drugs. Taken together, the current results suggest that the expression of Aven is regulated by the Akt signaling pathway through cathepsin D activity, which contributes to the sensitivity of cancer cells to chemotherapeutic agents.
\end{abstract}

\section{Introduction}

Cells respond to DNA damage by activating cell cycle checkpoints and DNA repair mechanisms or by engaging proapoptotic pathways (1-3). Genotoxic chemotherapeutic agents target DNA in order to activate mitochondrial apoptotic pathways in cancer cells $(4,5)$. Dysregulation of the DNA damage-induced apoptotic pathway triggers tumorigenesis and may lead to unwanted chemoresistance $(6,7)$. Therefore, it is important to identify the mechanisms of

Correspondence to: Professor Yong Kee Kim, Research Center for Cell Fate Control, College of Pharmacy, Sookmyung Women's University, 100 Cheongpa-ro 47-gil, Yongsan-gu, Seoul 140-742, Republic of Korea

E-mail: yksnbk@sm.ac.kr

Key words: Aven, apoptosis, Akt, cathepsin D resistance to DNA damage-induced apoptosis and to target these mechanisms in order to increase the effectiveness of cancer therapy.

Aven has been identified as an intracellular apoptosis inhibitor by interacting with B-cell lymphoma-extra large $(\mathrm{Bcl}-\mathrm{xL})$ and apoptotic protease activating factor 1 (Apaf-1) (8). Aven interacts with the N-terminal region and BH1 domain of Bcl-xL, which enhances the anti-apoptotic function of Bcl-xL (9). In addition, Aven interrupts Apaf-1 oligomerization, leading to inactivation of caspase- 9 and inhibition of apoptosis (8). Furthermore, it has been previously reported that Aven may be cleaved at L144/196 sites by the aspartic protease, cathepsin D (10). The cleaved C-terminal of Aven, $\Delta \mathrm{N}$-Aven, is essential for its anti-apoptotic function. In addition to its anti-apoptotic functions, Aven serves as a signal transducer in the ataxia telangiectasia mutated (ATM) activation pathway during DNA damage $(11,12)$. Activated ATM phosphorylates Aven on S135/308 and induces full activation of Aven. The activated Aven then further enhances ATM activation, leading to the activation of downstream pathway components to inhibit Cdc 25 activation and enhance Wee1/Myt kinase activity. This results in the subsequent inhibition of mitotic entry. Notably, it has been reported that the protein levels of Aven are increased in acute leukemias $(13,14)$. Aven overexpression was found to be associated with a poor prognosis in childhood acute lymphoblastic leukemia and may be useful as a novel prognostic indicator in this malignancy (13). These studies suggest a potential oncogenic role for the anti-apoptotic protein Aven. However, the molecular mechanism underlying the expression of Aven remains to be elucidated.

The present study described the regulation of Aven protein levels by the Akt signaling pathway and cathepsin D. Cellular levels of full-length Aven are maintained by proteolytic cleavage as a result of cathepsin D activity, which is associated with the Akt signaling pathway, rather than transcriptional control and protein stability. Higher expression of cathepsin D decreases levels of full length Aven, but increases $\Delta \mathrm{N}$-Aven levels in cancer cells, leading to the resistance of cancer cells to anticancer drugs and vice versa. The present results suggest that Aven level is regulated through the Akt signaling pathway by cathepsin D activity, which contributes to the sensitivity of cancer cells to chemotherapeutic agents. 


\section{Materials and methods}

Cells and reagents. MCF7 human breast cancer cell line (American Type Culture Collection, Manassas, VA, USA) was cultured in Dulbecco's modified Eagle's medium (Invitrogen Life Technologies, Carlsbad, CA, USA) supplemented with $10 \%$ fetal bovine serum (HyClone Laboratories, Logan, UT, USA) and $1 \%$ penicillin/streptomycin (Invitrogen Life Technologies). MCF7 constitutively active (CA)-Akt cells were established by stable transfection with pcDNA3 Myr HA Akt1 (\#9008; Addgene, Cambridge, MA, USA) into MCF7 cells. Cycloheximide (CHX), doxorubicin and 3-(4,5-dimethylthiazol-2-yl)-2,5-diphenyltetrazolium bromide (MTT) were purchased from Sigma-Aldrich (St. Louis, MO, USA). LY294002 was purchased from Biomol Research Labs (Farmingdale, NY, USA). All other reagents were obtained from Sigma-Aldrich, unless otherwise specified.

Plasmids and transfection. Human Aven cDNA was cloned into the pEGFP-C1 vector to generate GFP-Aven. The cells on $60 \mathrm{~mm}$ plates were transfected using TransIT-2020 (Mirus, Madison, WI, USA) according to the manufacturer's instructions. The cells were subcultured for $24 \mathrm{~h}$ prior to transfection on $60 \mathrm{~mm}$ plates. For use in transfection, $15 \mu \mathrm{l}$ TransIT-2020 was mixed with $5 \mu \mathrm{g}$ of plasmid and incubated for $30 \mathrm{~min}$ at room temperature. The TransIT-2020 reagent:DNA complexes were added dropwise into the wells and then incubated for $72 \mathrm{~h}$. Following transfection, cell lysates were collected and analyzed further.

Western blot analysis. Cells were lysed with $50 \mathrm{mM}$ Tris- $\mathrm{HCl}$ (pH 7.5), $120 \mathrm{mM} \mathrm{NaCl}, 20 \mathrm{mM} \mathrm{NaF}, 1 \mathrm{mM}$ EDTA, $5 \mathrm{mM}$ EGTA, $15 \mathrm{mM}$ sodium pyrophosphate, $30 \mathrm{mM}$ p-nitrophenyl phosphate, $1 \mathrm{mM}$ benzamidine, $0.1 \mathrm{mM}$ phenylmethylsulfonyl fluoride and $1 \%$ Nonidet P-40 for $20 \mathrm{~min}$ at $4^{\circ} \mathrm{C}$ and subsequently centrifuged at $15,000 \mathrm{x}$ g for $15 \mathrm{~min}$ at $4^{\circ} \mathrm{C}$. Cell lysates were boiled in Laemmli sample buffer for $3 \mathrm{~min}$, and $30 \mu \mathrm{g}$ protein was subjected to either 10 or $12 \%$ SDS-PAGE (Mini-Protean Electrophoresis system; Bio-Rad, Hercules, CA, USA) depending on the molecular weight of the target proteins. Proteins were then transferred to polyvinylidene difluoride membranes (Millipore Corporation, Billerica, MA, USA) according to standard procedures. Membranes were blocked in Tris-buffered saline containing $0.05 \%$ Tween 20 (M. Biotech, Hanam, South Korea) and 5\% non-fat dry milk for $1 \mathrm{~h}$. Following blocking, membranes were probed overnight at $4^{\circ} \mathrm{C}$ with primary antibodies in antibody dilution buffer (Tris-buffered saline containing $0.05 \%$ Tween 20 and $1 \%$ nonfat dry milk), followed by $1 \mathrm{~h}$ incubation with secondary antibodies at room temperature. Antibody detection was accomplished with WesternBright $^{\mathrm{TM}}$ ECL (Advansta Inc., Menlo Park, CA, USA) and exposed on Hyperfilm (Eastman Kodak Co., Rochester, NY, USA). The following primary antibodies were used: rabbit polyclonal immunoglobulin $\mathrm{G}$ (IgG) anti-green fluorescent protein (GFP; sc-8334; 1:1,000), rabbit polyclonal IgG anti-poly ADP ribose polymerase (PARP; sc-7150; 1:1,000) and rabbit polyclonal IgG anti-p21 (sc-756; 1:1,000) were purchased from Santa Cruz Biotechnology, Inc. (Dallas, TX, USA); rabbit polyclonal anti-pAkt (\#9271s; 1:1,000), rabbit polyclonal anti-Akt (\#9272s; 1:1,000), rabbit polyclonal anti-Aven (\#2300s; 1:1,000) and mouse monoclonal $\operatorname{IgG}_{2 \mathrm{a}}$ anti-pS6 (\#9206s; 1:1,000) obtained from Cell Signaling Technology, Inc. (Beverly, MA, USA); mouse monoclonal anti-cathepsin D (\#610800; 1:1,000) purchased from BD Biosciences (San Diego, CA, USA) and mouse monoclonal $\mathrm{IgG}_{1}$ anti- $\beta$-actin (A1978; 1:3,000) purchased from Sigma-Aldrich. Peroxidase-conjugated secondary antibodies polyclonal goat anti-rabbit IgG (\#111-035-003; 1:1,000) and polyclonal goat anti-mouse IgG (\#115-035-003; 1:1,000) were obtained from Jackson Immunoresearch Laboratories, Inc. (West Grove, PA, USA).

Determination of RNA and protein stability. Single-stranded cDNA was made with reverse transcriptase (M. Biotech, Hanam, South Korea) from $1 \mu \mathrm{g}$ of RNA using an oligo-(dT) $)_{18}$ primer. Subsequently, reverse-transcription quantitative polymerase chain reaction (RT-qPCR) analysis was performed to determine the relative expression levels of the Aven and GAPDH genes. PCR was performed for 35 cycles with an annealing temperature of $55^{\circ} \mathrm{C}$. PCR products were analyzed by electrophoresis on agarose gels.

Protein stability assay. For the protein stability analysis, the cells were treated with $10 \mu \mathrm{g} / \mathrm{ml} \mathrm{CHX}$ and then whole cell lysates were prepared at $0,0.25,0.5,1,3,6,12$ and $24 \mathrm{~h}$. The lysates $(50 \mu \mathrm{g})$ were then subjected to western blot analysis to identify the Aven and $\beta$-actin proteins.

MTT assay. The MTT viability assay was performed with slight modifications as previously described $(15,16)$. MTT was first prepared as a stock solution of $5 \mathrm{mg} / \mathrm{ml}$ in phosphate-buffered saline (PBS; pH 7.2) and was filtered. Briefly, cells (200 $\mu \mathrm{l} /$ well) were seeded at $3 \times 10^{4}$ cells $/ \mathrm{ml}$ into 96 well plates in the presence of various concentrations $(0.005-1 \mu \mathrm{M})$ of doxorubicin (or vehicle control) for $72 \mathrm{~h}$. MTT was added to the cultures during the last $4 \mathrm{~h}$ of incubation and then the media was removed with a needle and syringe. The blue formazan crystals trapped in cells were dissolved in sterile dimethyl sulfoxide $(100 \mu \mathrm{l})$ by incubating at room temperature for $30 \mathrm{~min}$. The well plate was read using the Epoch Microplate Spectrophotometer ${ }^{\text {TM }}$ (BioTek Instruments, Inc., Winooski, VT, USA) with absorbance at a wavelength of $570 \mathrm{~nm}$ with a reference wavelength of $630 \mathrm{~nm}$.

\section{Results}

Expression of Aven is regulated through the Akt signaling pathway. To identify the molecular mechanism involved in Aven expression, the expression level of Aven in several cell lines was analyzed. Notably, the expression level of Aven in MCF7 CA-Akt cell lines was significantly higher than that of its parental MCF7 cells (Fig. 1A). To confirm the possible involvement of the Akt signaling pathway in Aven expression, LY294002, an upstream kinase inhibitor of Akt, was administered to MCF7 CA-Akt cells. As shown in Fig. 1B, Aven expression was significantly abrogated by LY294002 treatment, suggesting that the expression level of Aven is associated with the Akt signaling pathway. In addition, this hypothesis was further supported by the observation that ectopic expression of the constitutively active form of Akt significantly increases the level of Aven in MCF7 cells, but its dominant negative form does not (Fig. 1C). 

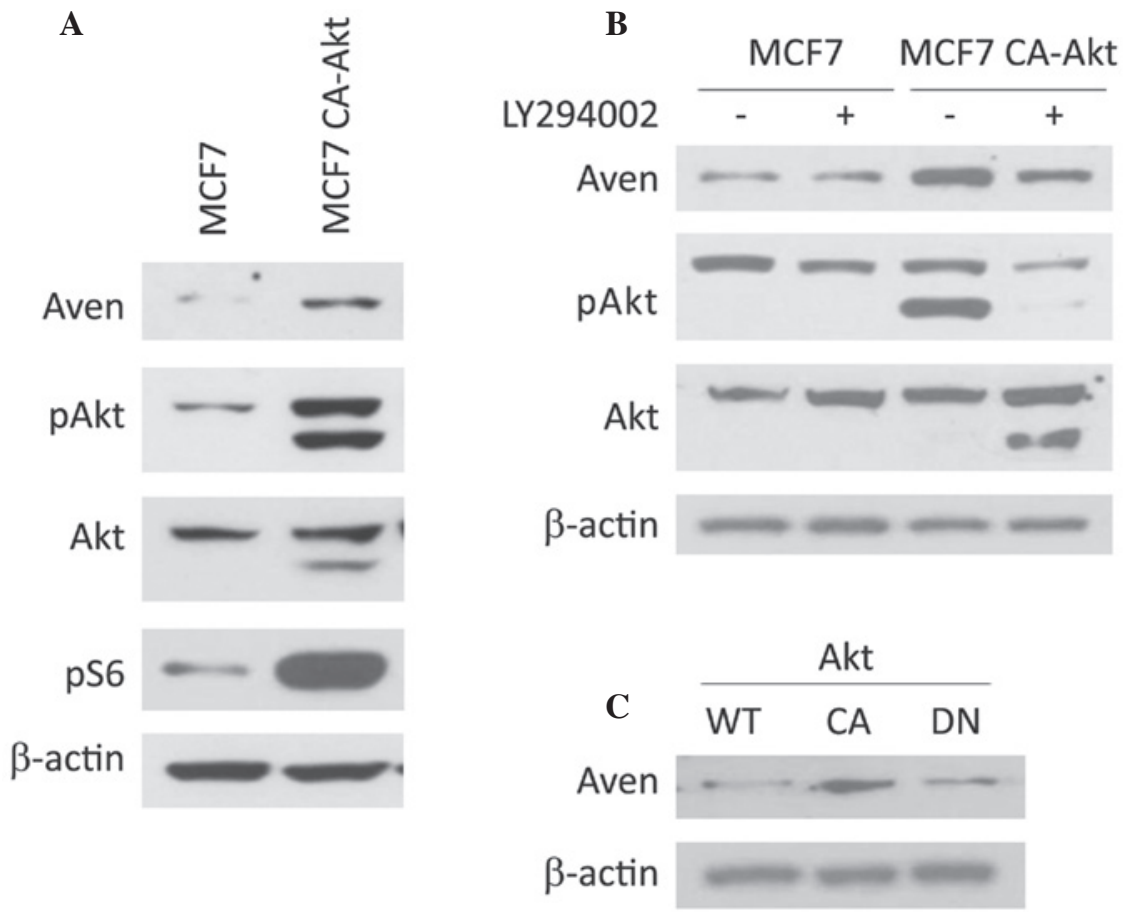

Figure 1. Involvement of the Akt signaling pathway in the expression of Aven. (A) Comparison of Aven expression in MCF7 and MCF7 CA-Akt cell lines. (B) Effect of LY294002 on Aven expression. Cells were treated with LY294002 $(20 \mu \mathrm{M})$ for $12 \mathrm{~h}$ and then the levels of phosho-Akt and phospho-S6 were analyzed by western blotting. (C) MCF7 cells were transiently transfected with expression vectors encoding Akt genes. After 3 days, cells were harvested and then cell lysates were immunoblotted with the indicated antibodies. WT, wild type; CA, constitutively active form; DN, dominant negative form.

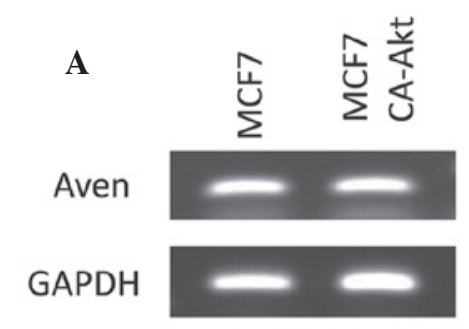

B

MCF7

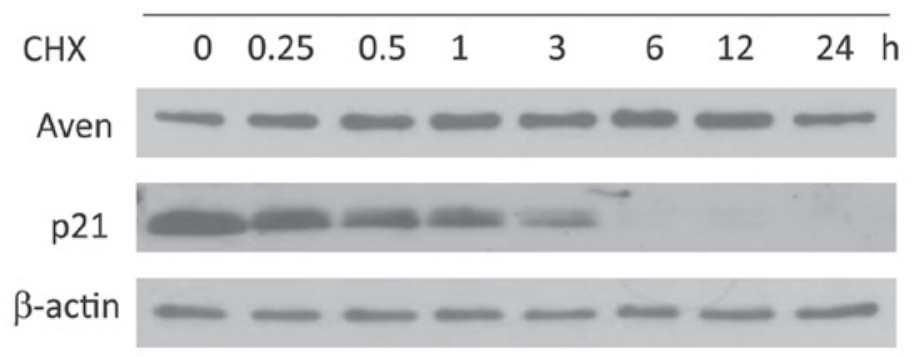

Figure 2. Aven expression is not affected by its transcription level or protein stability. (A) mRNA expression levels of Aven in MCF7 and MCF7 CA-Akt cells were determined by reverse transcription-quantitative polymerase chain reaction. (B) Analysis of Aven protein stability in MCF7 cells. Cells were treated with CHX $(10 \mathrm{mg} / \mathrm{ml})$ for the indicated time periods and cell extracts were analyzed by western blotting. CA, constitutively active; CHX, cycloheximide.

Expression of Aven is not affected by transcriptional control or protein stability. To elucidate whether the level of Aven may be regulated by a transcriptional process, the mRNA level of Aven was analyzed in MCF7 and MCF7 CA-Akt cells. As shown in Fig. 2A, no significant difference was observed in the mRNA levels of Aven between these two cells, which was further confirmed by qPCR (data not shown). This finding indicates that accumulation of Aven in MCF7 CA-Akt cells is regulated in a transcription-independent manner. Subsequently, the degradation rate of the Aven protein in MCF7 cells was determined using western blot analysis along a time course following addition of the protein synthesis 


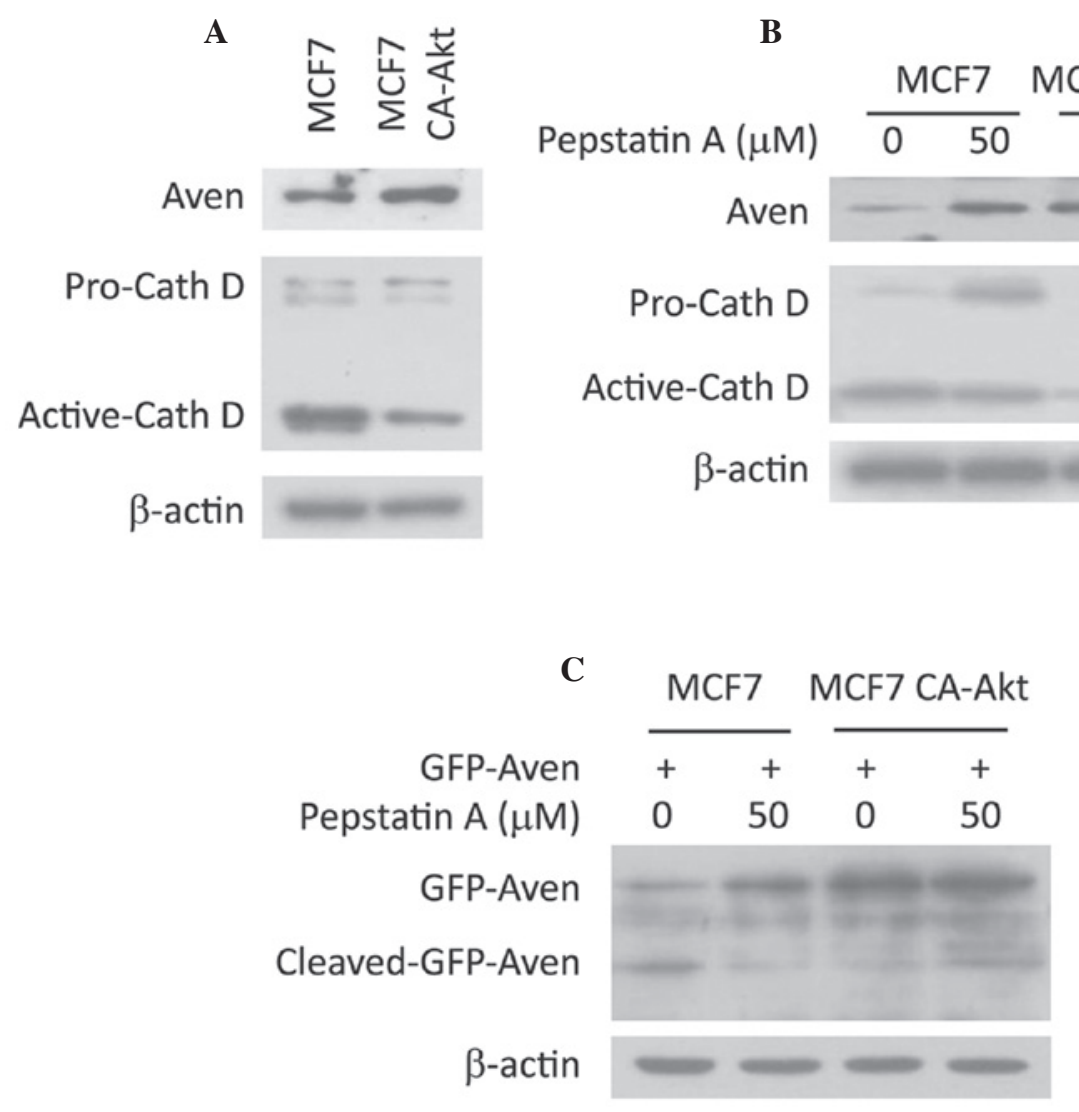

Figure 3. Involvement of cathepsin D in maintaining full-length Aven level. (A) Comparison of cathepsin D and Aven expression in MCF7 and MCF7 CA-Akt cell lines. (B) Effect of pepstatin A on Aven expression. Cells were treated with pepstatin A for $24 \mathrm{~h}$ and then cell extracts were analyzed by western blotting. (C) MCF7 and MCF7 CA-Akt cells were transfected with GFP-Aven for $48 \mathrm{~h}$ and then treated with pepstatin A for $24 \mathrm{~h}$. The level of Aven from cell extracts was detected using western blotting with GFP antibody. CA, constitutively active; GFP, green fluorescent protein.

inhibitor, cycloheximide. As shown in Fig. 2B, Aven protein stability was not significantly altered up to $24 \mathrm{~h}$ (Fig. 2B), indicating that Aven is a stable protein in cellular systems. In addition, the protein level of Aven in MCF7 CA-Akt cells was not altered during the $24 \mathrm{~h}$ time period (data not shown). All these results suggest that accumulation of Aven in MCF7 CA-Akt cells may not be affected by its stability.

Cathepsin D is responsible for maintaining the levels of full-length Aven. A previous study revealed that full-length Aven may be cleaved by cathepsin D to unleash its anti-apoptotic potential (10). There are two cleavage sites between amino acid 144/145 and between amino acid 196/197 in the Aven protein. Therefore, it was investigated whether cathepsin D regulates the level of Aven. As shown in Fig. 3A, the expression level of cathepsin D in MCF7 CA-Akt cells was lower than that of MCF7 cells, which was inversely correlated with Aven levels, indicating that the expression of cathepsin D may be a major determinant for full-length Aven level in MCF7 CA-Akt cells. To confirm this hypothesis, Aven levels were compared in the absence or presence of pepstatin A, an inhibitor of cathepsin D. As shown in Fig. 3B, Aven levels were markedly increased following treatment with pepstatin A, supporting the possible role of cathepsin D in the regulation of Aven levels. Subsequently, the present study aimed to detect cleaved Aven fragment, which is generated by cathepsin D activity in MCF7 cells, however, the Aven antibody was not able to detect the fragment (data not shown). Thus, a GFP-Aven overexpression system was used. When GFP-Aven was overexpressed in MCF7 cells, full-length GFP Aven and cleaved GFP-Aven were clearly detected. However, the only form of GFP-Aven in MCF7 CA-Akt cells was full-length and the cleaved form was not detected (Fig. 3C). In addition, pepstatin A treatment resulted in an evident increase in the full-length form and a decrease in the cleaved form (Fig. 3C). These findings support the hypothesis that the cellular level of full-length Aven is determined by cathepsin D activity, which may be regulated through the Akt signaling pathway.

Cleaved-Aven affects drug resistance. It has previously been demonstrated that $\Delta \mathrm{N}$-Aven cleaved by cathepsin $\mathrm{D}$ is the active form for the anti-apoptotic functions of Aven (10). Thus, the present study aimed to determine whether the expression levels of Aven affect the sensitivity of cancer cells to chemotherapeutic agents. The present data demonstrate that MCF7 CA-Akt cells are more sensitive to doxorubicin than the parental MCF7 cells, which was evidenced by a cell proliferation assay (Fig. 4A) and PARP cleavage (Fig. 4B). These results suggest that higher levels of $\Delta \mathrm{N}$-Aven may contribute to doxorubicin resistance. To further clarify this theory, the effect of pepstatin A on doxorubicin-induced apoptosis in MCF7 cells was determined. As shown in Fig. 4C, pepstatin A treatment led to an increase in doxo- 


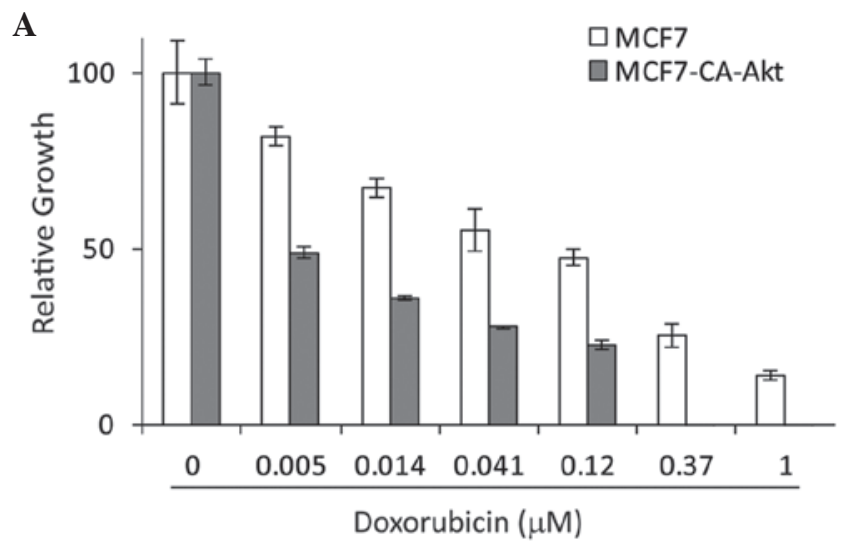

B

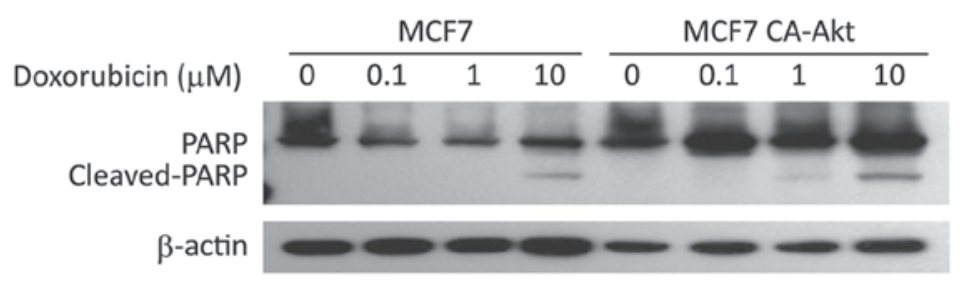

C

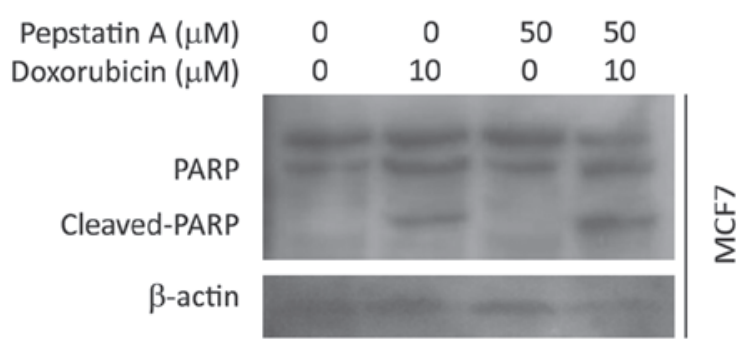

Figure 4. Effects of $\triangle \mathrm{N}$-Aven levels on drug resistance. (A) Sensitivity of MCF7 and MCF7 CA-Akt cells to doxorubicin was examined by 3-(4,5-dimethylthiazol-2-yl)-2,5-diphenyltetrazolium bromide analysis following incubation of cells with the indicated concentrations of drugs. (B) MCF7 and MCF7 CA-Akt cells were treated with doxorubicin for $24 \mathrm{~h}$ and cell extracts were analyzed by western blotting. (C) MCF7 cells were treated with pepstatin A for $24 \mathrm{~h}$ and then with doxorubicin. Total cell extracts were analyzed by western blotting. Cleavage of PARP was detected following doxorubicin treatment. CA, constitutively active; PARP poly ADP ribose polymerase.

rubicin-induced PARP cleavage. These results indicate that inhibition of $\triangle \mathrm{N}$-Aven formation by pepstatin $\mathrm{A}$ is associated with increased apoptosis in MCF7 cells and full-length Aven may be insufficient to inhibit apoptosis.

\section{Discussion}

Drug resistance to chemotherapy is a major obstacle for the treatment of several types of tumor and is an important cause of cancer chemotherapy failure. The cellular resistance of cancer cells to chemotherapeutic agents may be divided into two types of resistance; intrinsic resistance and extrinsic resistance $(17,18)$. Intrinsic resistance is predominantly involved in cell differentiation or in genetic alterations during tumor initiation. Extrinsic resistance results from the expansion of rare genetic variants in a tumor cell population. A good example is multidrug resistance (MDR) in which cells are resistant to numerous structurally and functionally unrelated chemotherapeutic agents $(19,20)$. Several molecular mechanisms have been proposed to explain MDR, including tumor cell-specific mechanisms such as decreased cellular drug accumulation, drug sequestration into intracellular vesicles, DNA repair pathway activation, which counteracts the effects of the drugs and evasion of apoptosis or cell cycle arrest (21-23). In addition, genes that control cell death and survival signaling, including the genes encoding B-cell lymphoma 2 and p53, may acquire mutations that lead to drug resistance through modulation or impairment of apoptosis. In addition, activation of alternative signaling pathways that modulate cell migration, proliferation and apoptosis may be involved in the development of drug-resistance pathways $(24,25)$.

Aven has previously been reported to act as an intracellular anti-apoptotic molecule through regulating Bcl-xL and Apaf-1 (8,9). Additionally, Aven may be cleaved at L144/196 sites by aspartic protease cathepsin D and the cleaved C-terminal of Aven, $\Delta \mathrm{N}$-Aven, is essential for its anti-apoptotic function (10). These findings indicate that intracellular levels of Aven may contribute to drug resistance to chemotherapeutic agents. The current data reveal that accumulation of full length Aven occurred through the Akt signaling pathway (Fig. 1). In addition, this accumulation was associated with inhibiting its proteolytic cleavage by cathepsin D (Fig. 3), indicating that the Akt signaling pathway may be associated with the anti-apoptotic function of Aven. 
It has been well demonstrated that the Akt signaling pathway is associated with tumor cell survival, proliferation and invasiveness $(19,26)$. The activation of Akt is also one of the most frequent alterations observed in human cancer and tumor cells. MCF7 CA-Akt cells that are constantly active grow more rapidly than their parental MCF7 cells (data not shown). However, it was observed that MCF7 CA-Akt cells are more sensitive to chemotherapeutic agents (Fig. 4). Furthermore, inhibition of cathepsin D by pepstatin A resulted in an increase in sensitivity to chemotherapeutic agents (Fig. 4). These results clearly indicate that the ratio of $\Delta \mathrm{N}$-Aven to full length Aven is governed by cathepsin D, which is regulated by the Akt signaling pathway.

In conclusion, the present data demonstrate the regulatory mechanism underlying the expression of Aven through the Akt signaling pathway and cathepsin D activity. Akt signaling and cathepsin D activity are intracellular regulators for maintaining the ratio of $\Delta \mathrm{N}$-Aven to full length Aven, which contribute to resistance to chemotherapeutic agents.

\section{Acknowledgements}

This study was supported by a grant from the National Research Foundation of Korea funded by the Korean government (The Ministry of Science, ICT and Future Planning; grant nos. 2011-0030074 and 2012R1A1A2043451).

\section{References}

1. Rich T, Allen RL and Wyllie AH: Defying death after DNA damage. Nature 407: 777-783, 2000.

2. Roos WP and Kaina B: DNA damage-induced cell death by apoptosis. Trends Mol Med 12: 440-450, 2006.

3. Bernstein C, Bernstein H, Payne CM and Garewal H: DNA repair/pro-apoptotic dual-role proteins in five major DNA repair pathways: fail-safe protection against carcinogenesis. Mutat Res 511: 145-178, 2002.

4. Herr I and Debatin KM: Cellular stress response and apoptosis in cancer therapy. Blood 98: 2603-2614, 2001.

5. Sanchez-Prieto R, Rojas JM, Taya Y and Gutkind JS: A role for the p38 mitogen-acitvated protein kinase pathway in the transcriptional activation of p53 on genotoxic stress by chemotherapeutic agents. Cancer Res 60: 2464-2472, 2000.

6. Okada $\mathrm{H}$ and Mak TW: Pathways of apoptotic and non-apoptotic death in tumour cells. Nat Rev Cancer 4: 592-603, 2004.

7. Maddika S ande SR, Panigrahi S, et al: Cell survival, cell death and cell cycle pathways are interconnected: implications for cancer therapy. Drug Resist Updat 10: 13-29, 2007.
8. Chau BN, Cheng EH, Kerr DA and Hardwick JM: Aven, a novel inhibitor of caspase activation, binds Bcl-xL and Apaf-1. Mol Cell 6: 31-40, 2000.

9. Kutuk O, Temel SG, Tolunay S and Basaga H: Aven blocks DNA damage-induced apoptosis by stabilising Bcl-xL. Eur J Cancer 46: 2494-2505, 2010.

10. Melzer IM, Fernandez SB, Bosser S, et al: The Apaf-1-binding protein Aven is cleaved by Cathepsin D to unleash its anti-apoptotic potential. Cell Death Differ 19: 1435-1445, 2012.

11. Guo JY, Yamada A, Kajino T, et al: Aven-dependent activation of ATM following DNA damage. Curr Biol 18: 933-942, 2008.

12. Esmaili AM, Johnson EL, Thaivalappil SS, Kuhn HM, Kornbluth S and Irusta PM: Regulation of the ATM-activator protein Aven by CRM1-dependent nuclear export. Cell Cycle 9: 3913-3920, 2010.

13. Choi J, Hwang YK, Sung KW, et al: Aven overexpression: association with poor prognosis in childhood acute lymphoblastic leukemia. Leuk Res 30: 1019-1025, 2006.

14. Eissmann M, Melzer IM, Fernandez SB, et al: Overexpression of the anti-apoptotic protein AVEN contributes to increased malignancy in hematopoietic neoplasms. Oncogene 32: 2586-2591, 2013.

15. Mosmann T: Rapid colorimetric assay for cellular growth and survival: application to proliferation and cytotoxicity assays. J Immunol Methods 65: 55-63, 1983.

16. Jo SK, Hong JY, Park HJ and Lee SK: Anticancer activity of novel daphnane diterpenoids from daphne genkwa through cell-cycle arrest and suppression of Akt/STAT/Src signalings in human lung cancer cells. Biomol Ther (Seoul) 20: 513-519, 2012.

17. Fulda $\mathrm{S}$ and Debatin KM: Extrinsic versus intrinsic apoptosis pathways in anticancer chemotherapy. Oncogene 25: 4798-4811, 2006.

18. Gottesman MM: Mechanisms of cancer drug resistance. Annu Rev Med 53: 615-627, 2002.

19. Fresno Vara JA, Casado E, de Castro J, Cejas P, Belda-Iniesta C and Gonzalez-Baron M: PI3K/Akt signalling pathway and cancer. Cancer Treat Rev 30: 193-204, 2004.

20. Szakacs G, Paterson JK, Ludwig JA, Booth-Genthe C and Gottesman MM: Targeting multidrug resistance in cancer. Nat Rev Drug Discov 5: 219-234, 2006.

21. Krishna R and Mayer LD: Multidrug resistance (MDR) in cancer. Mechanisms, reversal using modulators of MDR and the role of MDR modulators in influencing the pharmacokinetics of anticancer drugs. Eur J Pharm Sci 11: 265-283, 2000.

22. Lothstein L, Israel M and Sweatman TW: Anthracycline drug targeting: cytoplasmic versus nuclear - a fork in the road. Drug Resist Updat 4: 169-177, 2001.

23. Stavrovskaya AA: Cellular mechanisms of multidrug resistance of tumor cells. Biochemistry (Mosc) 65: 95-106, 2000.

24. El Maalouf G, Le Tourneau C, Batty GN, Faivre S and Raymond E: Markers involved in resistance to cytotoxics and targeted therapeutics in pancreatic cancer. Cancer Treat Rev 35: $167-174,2009$.

25. Tamburrino A, Piro G, Carbone C, Tortora G and Melisi D: Mechanisms of resistance to chemotherapeutic and anti-angiogenic drugs as novel targets for pancreatic cancer therapy. Front Pharmacol 4: 56, 2013.

26. Altomare DA and Testa JR: Perturbations of the AKT signaling pathway in human cancer. Oncogene 24: 7455-7464, 2005. 